\title{
A mockup stochastic program to study the impact of design uncertainty on ETO shipbuilding planning
}

\author{
Hajnalka Vaagen \\ SINTEF Technology and Society, Department of Applied Economics, SP. Andersensvei 5, \\ 7432 Trondheim, Norway, hajnalka . vaagen@sintef . no \\ Michal Kaut \\ SINTEF Technology and Society, Department of Applied Economics, SP. Andersensvei 5, \\ 7432 Trondheim, Norway, michal kaut@sintef.no
}

\begin{abstract}
A major driver of planning complexity in dynamically changing ETO shipbuilding is design uncertainty far into the design planning and production processes. This leads to uncertainty in task and project completion time, and complex dependencies and correlations driven by the uncertainty in multiple task parameters. The problem is difficult to be solved exactly, and decisionmaking is largely based on experience and gut feeling, with subsequent behavioral challenges. We build a mockup stochastic program to draw attention toand analyze the complexity of formulating and solving the engineering design planning problem. We demonstrate how design uncertainty is affecting the planning complexity and solutions.
\end{abstract}

Keywords: ETO, project planning, stochastic programming

\section{Problem description and relevant literature}

A major driver of planning complexity in customized dynamically changing shipbuilding projects is design uncertainty through the engineering design- and far into the production processes. This is leading to continuous adjustments in planning, procurement and execution [1], and defines the uncertainty to be dealt with on a daily basis. Design uncertainty generates uncertainty in the technical information and completion time for a chain of connected tasks, and negative/positive correlations. One design alternative potentially excludes other alternatives (e.g. weight \&space restrictions), and may lead to negative correlations between the uncertain activities. Uncertain activities competing for the same resources are other sources of negative correlations. For more on project correlations see [7]. In summary, we deal with a planning problem with two-level uncertainty, where stochastic task completion times are conditioned by uncertain design, and correlations driven by the uncertainty in multiple task parameters. Minimal use of resources and reliable adherence to tight schedules is challenging here. Creating flexibility by solving the true planning com-

adfa, p. 1, 2011.

(C) Springer-Verlag Berlin Heidelberg 2011 
plexity is obviously difficult. Behavioural and organizational issues are other reasons for lack of flexibility in plans. Investments in flexibility normally have costs early and potential income late, and hence often undervalued by the decision makers. Randomness is also often lost between departments (e.g. between sales and engineering), resulting in 'reduced' randomness and reduced value of flexibility. Many solutions ignore, as such, important characteristics of the true planning problem, and lack the necessary flexibility [8]. As practitioners increasingly recognize the shortcomings, model-based decision aids are often replaced by judgmental processes [8] that automatically open up for behavioral challenges. Judgmental decision processes are suggested to work - in an ad-hoc manner though - in project organizations built on trust and experience [8]. Dealing with the described complexity judgmentally is, however, not less complex; e.g. understanding how activities are correlated with each other is important for prioritization of the project tasks, but often biased by the limitations in working memory when dealing with complex issues simultaneously, natural risk aversion, invisible \& illusory correlations, etc. We believe hence the decision processes to benefit from an intensified analytical approach, as models are logical to the givens. Stochastic programming explicitly models the value of future decisions that are made after the uncertainty is revealed (i.e. future choice on design alternatives), and might be a good approach despite complexity. Although buffer management is common to hedge against uncertainty, stochastic solutions are not "something plus the original solution". This is rather intuitive if we consider cases like adapting from an originally planned 'offshore cable-layer system' to a 'firefighter system' far into the production processes.

Hence, the motivation to build a mockup stochastic program to draw attention to- and analyze the complexity of formulating and solving ETO planning problems with complex uncertainty patterns, and to investigate what we can learn about the impact of design uncertainty on planning by analyzing small model instances.

The remaining of this section discusses chosen literature, with focus on uncertainty and correlations in project management and scheduling. The applied stochastic modeling approach is described in Section 2. The mockup design-engineering planning test case is implemented in Section 3, and concluded in Section 4.

Decision-making trends in project management and advances in scheduling techniques are presented in [6]; emphasizing the need for analytical approaches and risk handling competences to rapidly adapt changes. Best practice and shortcomings in different research streams on robust planning and decision-making in engineering construction projects are discussed in [8]. Scheduling methods like the critical path method algorithm CPM [5], balancing time and cost while resource-oriented, and the rather similar PERT, with the main difference on stochastic activity durations, have been in focus from the late fifties. PERT, and other simulation approaches provide a picture on project risk and simulate the effects of options for decision (before decision). These facilitate better planning, but still lack decisions. Most decision makers still choose the decisions that fit the most likely outcome, and overlook the potentially high costs of adapting to a different scenario. Attempts to overcome these shortcomings exist by adding decisions within the simulation model: e.g. the decision of in- 
creasing resources if we are late relative to the plan. Future decisions, such as the decision on a design alternative, are however not explicitly taken into account, as this cannot be done within a simulation model. The authors in [2] emphasize that even finding a way to formulate the general stochastic dynamic scheduling problem seems tedious, and that the problem remains unsolved.

The difficulties in modeling and solving large mixed integer stochastic problems also motivates academics to discuss whether uncertainty should be ignored in the planning, or when in the planning process should uncertainty be included and how. The authors in [3] provide an early discussion on the use of deterministic solutions in stochastic setting. By a mixed integer stochastic commodity flow example, the authors show that deterministically chosen edges are a good start in some cases, for the first stage stochastic integer (mixed-integer) problem, with a simple linear stochastic model to set capacities for the second stage. The planning case of this paper can be seen as a related scheduling problem, and it may be tempting to look at the 'deterministic skeleton' approach. We deal however with a two-level uncertainty pattern, where we anticipate deterministically chosen sequences not to deliver good solutions for different design alternatives.

The literature on correlations in project schedules is limited, mainly due to the analytical models' limitations in handling complex uncertainty and dependency patterns. Numerical stochastic programming approaches show to offer the ability to handle complicated distributions [9,3]. The authors in [10] formulate a product portfolio problem with bimodal distributions and complex dependency patterns, and show that hedging is mainly driven by the two possible design states (preferred /not preferred by the market), and not very sensitive to the specific values of the correlations or the marginal demand distributions of a particular design. It also shows high value in pairing items that are negatively correlated and at the same time substitutable. These findings apply to the case problem on a conceptual level, in that both of them treat a twolevel stochastics problem with complex dependencies. The authors in [4] model a stochastic network design problem, and suggest that by consolidating two negatively correlated demand flows, an effective use of capacities can be achieved. In cases with strong positive correlation between high-probability high-demands, the authors suggest schedules that accommodate the most probable scenarios with most demands being high at the same time. Network flexibility has low value in this positive correlation case, and resources are suggested to be used on planned outsourcing. These findings provide interesting insights, and show that correlations matter; a conclusion also supported by [11], claiming by the use of a simulation model that correlations may be more important than the distribution representing the task duration uncertainty.

\section{The applied stochastic modelling approach}

The problem to be modeled is a complex and unsolved two-level uncertainty planning problem. To better understand the challenges, we divide the modelling process into two separated steps. This paper models the higher level uncertainty - the stochastic design-, while keeping the design-dependent task durations deterministic. The aim 
is to say something useful on how design uncertainty is affecting the planning complexity and solutions. In a second step, not provided in this paper, the full two-level uncertainty will be modeled, with stochastic activity durations constrained by the higher level design uncertainty, and correlations between the stochastic activities.

Unfortunately, the full formulation of the stochastic model is out of the scope of this paper. Instead, we describe the applied modelling approach. We use the so-called compact formulation of a stochastic program, where all variables are directly indexed on the nodes of the scenario tree-as opposed to the scenario-based formulation, where variables are indexed by time and scenario and the tree structure is enforced using the so-called nonanticipativity constraints.

In each node $n$ of the scenario tree, we define the following binary variables for each activity $a$ :

$x_{-}(n, a) \quad$ has activity $a$ started at the start of node $n$ ?

$y_{-}(n, a) \quad$ has activity $a$ finished at the end of node $n$ ?

$z_{-}(n, a) \quad$ has activity $a$ been finished by the start of node $n$ ?

$u_{-}(n, a) \quad$ is activity $a$ running during node $n$ ?

$v_{-}(n, a) \quad$ has activity $a$ been stopped at start of node $n$ ?

In addition, we have continuous variables tracking resource usage at each node. From a conceptual point of view, it is important to realize that the only 'real' decision variables are $x_{\_}(n, a)$ and $u \_(n, a)$; the rest are auxiliary variables whose values are completely determined by others.

With these variables, we can implement the following functionality from the original problem description:

- Activity dependencies of type \{and\} (wait for all the specified activities) and \{or\} (wait for at least one activity).

- Stochastic dependencies: like above, but the dependency sets can differ between the scenario-tree nodes. This implements the stochastic design changes.

- An ongoing activity can be stopped at any time.

- Undo-activities: if activities $A$ and $B$ represent two different designs for the same element and we start work on $A$, we can require the use of a special activity that undoes $A$, before we can start working on $B$. Moreover, the duration of the undoactivity depends on the time spend on $A$.

- Resource usage per activity and period, to model access to labour, space, and equipment.

- Piece-wise linear resource-usage costs, to model higher costs for extra resources.

\section{Test case and model implementation}

\subsection{The mockup engineering-design problem}

The mockup engineering-design planning problem is illustrated by Figure 1. Real tasks are depicted by ellipses, indicator tasks with and-dependency by diamonds, or- 
dependency by rectangles, and the stochastic dependency by a combination of the two.

Assume a major outfitting structure with two possible design states $A$ and $B$, composed by two tasks piping $P$ and electro $D$; $(P A, D A)$ and $(P B, D B)$ respectively. These can be built in one stage and in two-stages, where the first stage decisions $P 1$ and $D 1$ can be used for both designs $A$ and $B$. The "wrong" design decision implies 'undo' activities after the design uncertainty is revealed, to remove the tasks from the 3D model and from the physical unit if the task is released in production. The duration and costs of this undo-activity depend on the time spent on the "wrong" design.

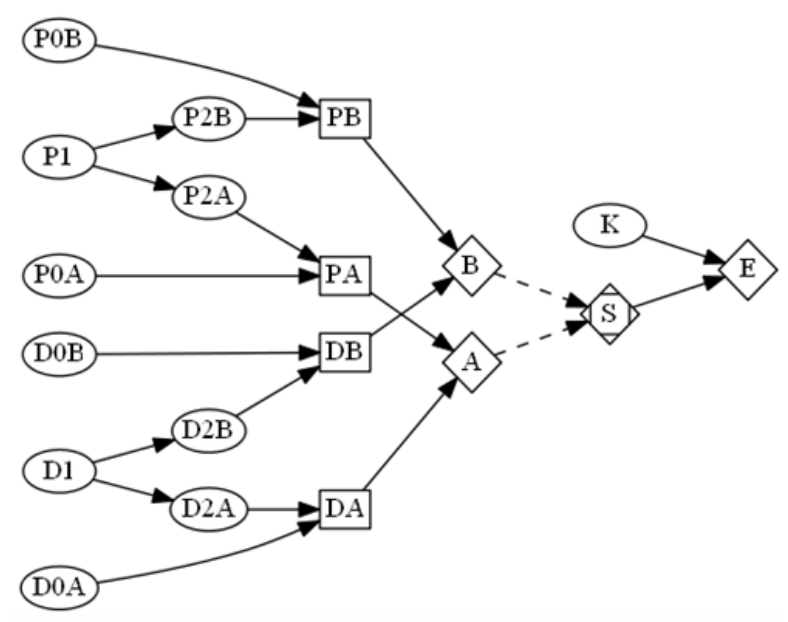

Fig. 1. Mockup engineering-design planning problem. Real tasks are depicted by ellipses, indicator tasks with and-dependency by diamonds, or-dependency by rectangles, and the stochastic dependency by a combination of the two.

\subsection{Test cases}

The following test cases are implemented and analysed. Case $\mathbf{1}$ is the deterministic static case, where we know ahead of time the preferred design alternative to be $A$ (or we assume we know) and we fix all decisions by the start. Case $\mathbf{2}$ is a deterministic dynamic case, where we plan for design $A$ but the world turns to be $B$, and we 'update' the plan during the planning horizon by fixing the deterministic solution for the deterministic scenario. Case $\mathbf{3}$ is the stochastic dynamic case, with uncertainty in design alternatives $A$ and $B$. The order of decisions is not fixed here, but depends on previous decisions and the realization of the random design.

The model has been written in the GNU MathProg modeling language and the test instances were solved using Fico(trademark) Xpress Optimizer v. 26.

Important model parameters applied in the analysis are as follows. In each leaf of the scenario tree the probability of design $A$ is $90 \%$ and $10 \%$ the alternative $B$. That is, the total probability of the first scenario is $0.9^{4}=0.6561$. The scenario tree - with information on the preferred alternative revealed at different nodes - is given by Figure 
2. Design alternative $A$ is preferred with probability 0.6561 in the scenario finishing in node 30 . In the other scenarios, information on the preferred design alternative $B$ arrives at different nodes (the scenario finishing in node 55, with prob $=0.1$; scenario finishing in node75 with prob $=0.09$; scenario finishing in node 90 , with $p r o b=0.081$; and scenario finishing in node 105 with prob $=0.0729$ )

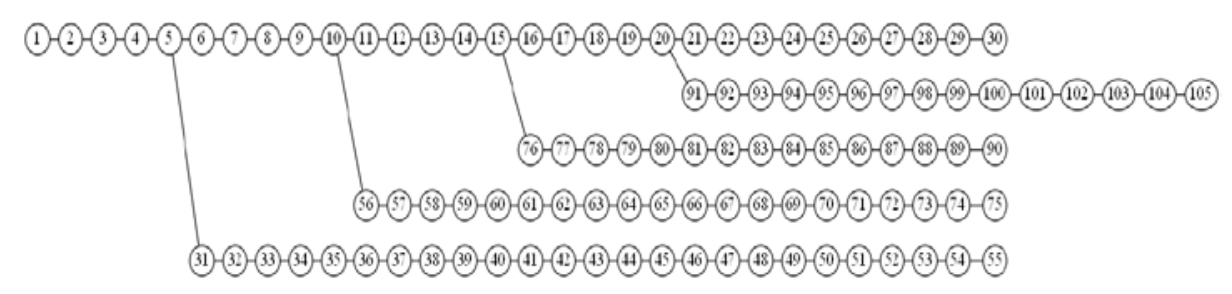

Fig. 2. The scenario tree

We consider a 'lookahead' planning horizon (usually 6-8 week of 5 days; days defined as periods in the model). Each activity uses 1 unit of 'labour' per period. The resource-usage costs are piece-wise linear, with 1.0 for the first two units used, 1.5 for the third and 2.0 for the fourth unit; reflecting the scarcity of engineering capacity. All activities related to design $B$ use one extra resource, with capacity of 1 . In other words, none of these activities can be performed in parallel. This resource is free. There is a penalty cost associated with each late finish, equaling $0.5 /$ period from period 16; 1.0 from period 21; 2.0 from period 26 and 4.0 from period 31. Activities have the following deterministic duration: $P_{0} A(10), P_{1}(7), P_{0} B(8), P_{2} A(4), P_{2} B(2), P A(0)$, $P B(0), D_{0} A(8), D 1(3), D_{0} B(10), D_{2} A(6), D_{2} B(8), K(10)$. Recall that for this paper we modeled a stochastic design/ deterministic task duration situation.

\subsection{Test results}

The optimal objective function value of the static deterministic Case 1 provides a lower bound of 29.000 on the total costs. This solution will, on average, be lower than the actual costs disregard the scheduling strategy applied [2]; i.e. Case 1 is an optimistic situation. Case 2, the dynamic variant of Case 1, analyses the adaptation patterns and costs from design $A$ to $B$. The total costs are 39.0712 ; an increase of $\mathbf{3 5 \%}$ from Case 1 . That is, decision makers believe to get the total cost of 29.000 , but in reality will end up 35\% higher; a potentially severe error. Obviously, an update like this is necessary to adapt new customer requirements. Recall also that the total cost is calculated by the use of engineering resources; a limited and critical resource for project completion time. In the model we allow for extra capacity for additional costs (e.g. overtime, flexible capacity), but this is not always the case. A substantial error in the planned capacity may lead to increase in completion time, a highly unwanted situation.

But if we don't know the preferred design alternative at the time of planning (and don't assume we know), what can we do before we learn which alternative is pre- 
ferred? The solution structure of the stochastic dynamic Case 3 shows a flexible hedging route, prepared to adapt both design alternatives, for a total cost of 36.008; i.e. decision makers can do $\mathbf{8 \%}$ better than if they adopt the deterministic reactive Case 2. This is the value of flexibility, i.e. the monetary value of the ability to make a decision later in time. The structure of the stochastic solution differs from the deterministic one in that the order of decisions is not fixed but depends on previous decisions and the realization of the random design variable. The solution prepares to adapt both design alternatives, by first scheduling tasks that can be used in both design alternatives, and secondly hedging by overlapping sequencing of both design specific tasks (where possible). This indicates that even when the probability of design $B$ is low ( $10 \%$ in each leaf of the scenario tree), hedging by preparing for both alternatives has value. In a deterministic setting hedging solutions are not wanted, as we know from the start that one of the 'investments' will be discarded. The IQ of hindsight is high. After the fact one of the deterministic solutions $A$ or $B$ will turn out to be best. Ahead of planning we don't know, though, which one will happen. And flexibility created by a proactive strategy allows for reduced adaptation costs.

Aside from showing the potential error of ignoring design uncertainty, the deterministic case provides an interesting finding, in that it prefers the two-stage design solution to the one-stage solution. Recall that both designs $A$ and $B$ can be constructed in one- or two-stages. Note that this specific behavior of the deterministic model is not driven by "flexibility" (as in a deterministic model all decisions are fixed and there is no flexibility), but to reduce the costs by more efficient allocation of the overlapping activities. A practical interpretation of this is concurrent engineering enabled by decomposing tasks into subtasks /modules that can be performed in different sequences. Concurrent engineering with overlapping activities that do not always follow the logic of sequential orders is highlighted by [1] and [8] as one important way to reduce completion times within the case context.

\section{Conclusion}

In this paper we formulated a stochastic dynamic model (a proactive strategy) to study the impact of design uncertainty on planning complexity and solutions, and demonstrated the value of flexibility. We show that good planning solutions for uncertain design are not a deterministic plan plus some slack on the top. Good solutions are flexible hedging strategies, where 'hedging' relates to being prepared for alternative design solutions, and 'flexible' refers to multi-step design solutions with the first step being part of both design alternatives. Although such flexible multi-step designs have extra development costs, the solutions enable adaptation to alternatives with least costs. This implies high value in early identifying design alternatives and activities that have most impact on the project completion time, and that often carries the most uncertainty and may require hedging plans in early phases.

We also demonstrate that the value of deterministic reactive strategies increases by concurrent engineering enabled through multi-step /modularized design. I.e. multistep design solutions have value also when an deterministic schedules are 'updated' 
according to new design requirements during the project execution. Concretely, we suggest that completion time is reduced by concurrent engineering enabled through module-based design; a finding that supports industrial state-of-practice.

Summarizing the paper, "Understanding why we need stochastic programs, being able to formulate them and finding out what it makes such solutions good, can help us to find good solutions without actually solving the stochastic programs."[3]. This paper is hence more than an attempt to formulate a complex and unsolved stochastic dynamic scheduling problem. It provides a case of applying small model instances to show the impact of design uncertainty on planning solutions. The insights derived potentially improve the judgmental abilities in planning dynamic and uncertain projects. In practices many companies need a planning guideline to understand where and when to develop flexibility and buffers.

Acknowledgements: This paper is part of the competence building research project NextShip, under Norwegian Research Council grant agreement 216418/O70.

\section{References}

1. Emblemsvag, J.: Lean project planning in shipbuilding. Journal of Ship Production and Design 30(2), 79-88 (2014).

2. Jørgensen, T., Wallace, S.W., 2000. Improving project cost estimation by taking into account managerial flexibility. European journal of Operational Research. 127, pp. 239-251.

3. King, A.J., Wallace, S.W.: Modeling with Stochastic Programming. Springer Series in Operations Research and Financial Engineering, Springer (2012)

4. Lium, A.G., Crainic, T.G., Wallace, S.W.: A study of demand stochasticity in stochastic network design. Transportation Science 43(2), 144\{157 (2009)

5. Morris, P.W.G.: The Management of Projects. Thomas Telford, London (1994).

6. Rolstadas, A., Pinto, J.K., Falster, P., Venkataraman, R.: Decision Making in Project management. NTNU Engineering Series, Fagbokforlaget (2014).

7. Schuyler, J.R.: Risk and Decision Analysis in Projects. Project Management Institute (2001)

8. Vaagen, H., Aas, B.: A multidisciplinary framework for robust planning and decisionmaking in dynamically changing engineering construction projects. In: Grabot, B., Vallespir, B., Gomes, S., Bouras, A., Kiritsis, D. (eds.) Advances in Production Management Systems. Innovative and Knowledge-Based Production Management in a Global-Local World. pp. 515\{522. Springer Berlin Heidelberg (2014), IFIP WG 5.7 International Conference, APMS 2014, Ajaccio, France, September 20-24, 2014.

9. Vaagen, H., Wallace, S.W.; Kaut, M., 2011. Modelling consumer-directed substitution. International Journal of Production Economics. 134(2) pp. 388-397.

10. Vaagen, H., Wallace S.W., 2008. Product variety arising from hedging in the fashion supply chains. International Journal of Production Economics 114(2), 431-455.

11. Wall, D.M., 2010. Distributions and correlations in Monte Carlo Simulation, Construction Management and Economics, 1997, 15:3, 241-258. 